\title{
GEOSPATIAL COMPUTATIONAL GRID FOR DIGITAL FORESTRY WITH THE INTEROPERABILITY
}

\author{
Guang Deng ${ }^{1, *}$, Xu Zhang ${ }^{1}$, Quoqing Li $^{2}$, Zhenchun Huang ${ }^{3}$ \\ ${ }^{1}$ Research Institute of Resource and Information Technique, Chinese Academy of Forestry, \\ Beijing, China, 100091 \\ ${ }^{2}$ China Remote Sensing Satellite Ground Station, China Academy of Science, Beijing, China, \\ 100086 \\ ${ }^{3}$ Department of Computer Science and Engineering, Tsinghua University, Beijing, China, \\ 100084 \\ * Corresponding author, Address: P.O. Box 33, Chinese Academy of Forestry, No. 1 \\ Dongxiaofu, Xiangshan Road, Beijing, 100091, P. R. China, Tel: +86-10-62889196, \\ Fax: +86-10-62872778, Email: dengg@caf.ac.cn
}

Abstract: In the Digital Forestry area, there is more and more requirements for spatial information connection and processing. From a prototype of geospatial computational grid (GCG), the relationship between the geospatial computational grid and OGC Interoperability protocols is proposed in this paper. A prototype of geospatial information grid is given in this paper. Digital Forestry Grid is proposed as a case of implementing of geospatial computational grid with OGC interoperability at the end of this paper.

Keywords: Geospatial Computational Grid; Digital Forestry; Interoperability

\section{INTRODUCTION}

Earth science is a data-intensive and computation-intensive scientific domain in which the applications always produce and analyze a large volume of distributed heterogeneous geospatial information. The grid concept is revolutionary, because it foresees the future integration of technologies to realize an observing and operating system with scalability and applicability over a broad range of earth surface phenomena. As forest 
management is a kind of earth resource science, we do the research on the Geospatial Computational Grid for forest management. In this paper, we discuss the relationship between the Geospatial Computational Grid and interoperability based the view of system construction.

As the amount of remote sensing imagery and related GIS database in the forest management area grows, there is an increasing problem of providing sufficient computational resources to assemble and analyze the datasets for environment science research or forestry affairs decision-making supporting.

Grid technology brings together geographically and organizationally dispersed computational resources, such as CPUs, storage systems, communication systems, data, software, instruments and human collaborators, which securely provide advanced distributed high-performance computing to users in one or more Virtual Organizations (VOs) (Foster, et al., 2001; Booth, et al., 2004). The Globus Project proposes the Open Grid Service Architecture (OGSA), Open Grid Service Infrastructure (OGSI) for Globus 3.0 and the Web Service Resource Framework (WSRF) for Globus 4.0 as the guidelines and specifications for system design and implementation to build a geospatial computational Grid (Brunett, 2001; Moore, et al., 1999; Karl, et al., 2004).

Although the grid technology can take an important role in geospatial computational grid design, but it is not enough because of the particularity of geospatial information. As follows, the description of the paper structure is given. First, we discuss the trends and problems which parallel and distributed computing for processing large-scale geospatial data would faced. Then, a prototype of geospatial information grid is given, which is a case study and a simple implementing of our understanding of geospatial computational grid. From this, we discuss the methods to integrate the geospatial interoperability to grid application. The Digital forestry Gird is the case of the methods discussed above. Finally, an opening conclusion is given for further research.

\section{A PROTOTYPE OF GEOSPATIAL COMPUTATIONAL GRID}

\subsection{Aims and structures}

A major goal of the geospatial computational grid is to make it as easy and transparent as possible for researchers to move jobs, GIS data and kinds of remote sensing data freely among the machines within the grid scope. The geospatial computational grid would consist of some resource provider (RP) 
sites. Each site connects through a dedicated geospatial data clearing house network, and provides high-end computing resources totaling more than specifically Floating Point Operations Per Second (FLOPS) of compute power and specifically available storage. Users have the option of storing their data, managing their jobs, and performing their computations on the machine most appropriate for their tasks, using grid technology for access to each resource.

A prototype system of geospatial computational grid is a spatial information grid which designed by our research team and has resolved many problems. By applying WSRF, the spatial information grid is constructed. Fig. 1 shows the structure of geospatial computational grid.
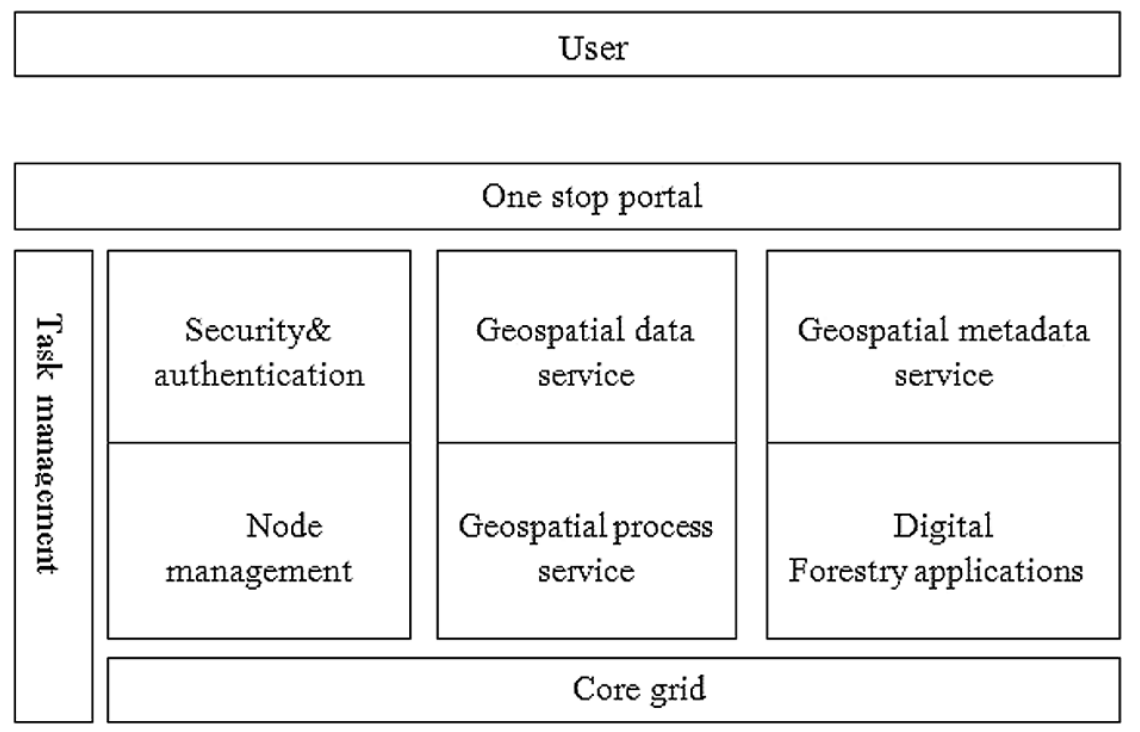

Fig. 1. The structure of geospatial computational grid

\subsection{Functions}

The geospatial computational grid provides many functional modules such as geospatial data processing and core grid management.

Node management is for monitoring and discovery of node for providing its information and its state, which also provide services through state registration, data modeling and local registry. By globe name service, the service in Geospatial Computational Grid could be understood. Task management is a Resource Allocation Manager for providing a common user interface for submitting a job to the dispersed multiple machines. The 
security and authentication function provides generic security services such as authentication, authorization and credential delegation for resources that will be run on the Grid. The geospatial metadata service and geospatial process service belongs to Metadata Catalog Service (MCS) for providing a mechanism for storing and accessing metadata of data and service.

Anyway the operational geospatial computational grid must deal with interoperability problems which involved in the related layers.

\section{INTEROPERABILITY AND GEOSPATIAL COMPUTATIONAL GRID}

\subsection{Protocols for interoperability}

The non profit, international, voluntary consensus standards organization, Open Geospatial Consortium, Inc. (OGC) is leading the development of interoperable standards for geospatial and location based services. In the geospatial community, the meaning of "interoperability" remains somewhat ambiguous, as do many of the benefits of "being interoperable". Therefore, the following interoperability mandate is suggested. To be interoperable, one should actively be engaged in the ongoing process of ensuring that the systems, procedures and culture of an organization are managed in such a way as to maximize opportunities for exchange and re-use of information and services, whether internally or externally (OGC, 2000; Vretanos, 2002; Cox, et al., 2003; Aktas, et al., 2004).

The successfully executed series of web-based geospatial interoperability initiatives, including Web Mapping Testbed (WMT) I, WMT II, and OGC Web Service Initiative (OWS) 1.1, and OWS 1.2 have produced a set of web-based data interoperability specifications, such as the OGC Web Mapping Service (WMS) specification which allows interactively assembling maps from multiple servers, the OGC Web Coverage Service (WCS) specification which provides an interoperable way of accessing geospatial data from multiple coverage servers, especially those data from remote sensing, and the OGC Catalogue Service-Web (CSW) specification which is based on e-business Registry Information Model (ebRIM) and aims to provide an object-oriented registry system for registering, managing and retrieval of geospatial resources, e.g. services, data and other objects (Chen, et al., 2005). 


\subsection{Agents for interoperability}

How to put OGC interoperable Web Service into the common computing grid is the key technologies to construct geospatial computational grid. We use the agents to integrate the interoperability and Geospatial Computational Grid. Table 1 shows the selected OpenGIS interoperable specification agents used in GCG. The basic methods to construct the agents can get from Li's paper (Li et al., 2005).

\section{DIGITAL FORESTRY GRID AS A CASE}

Digital Forestry Grid (DFG) is a kind of specialization application grid which supports for forest resource information management and forestry eco-construction projects. A characteristic of the DFG is in specialization grid resource management, business logic development and running engine of grid application. By grid transform of forestry resource information, DFG integrates the data resources of forest and forestry eco-construction projects in four levels (namely state, province, city and county). The data resources have kinds of types, such as remote sensing images, vector data, thematic attribute data and etc. Thematic grid services, such as data resource service, spatial information analysis service, online statistic computing service, is be developed independently, which realizes the sharing and usage of forestry information resources in deeply extents.

Using catalog interface service (Table 1), DFG find the wanted thematic forest resources data service. Using filter encoding and Web Feature Service (Table 1), the forest resources map can be set out on the web. (Fig. 2)

Table 1. OpenGIS interoperable specification agents for GCG

\begin{tabular}{lll}
\hline Acronym & Name & Agents at GCG Location \\
\hline CAT & Catalog Interface & Geospatial metadata service \\
CT & Coordinate Transformation Services & Geospatial data service \\
Filter & Filter Encoding & Geospatial data service \\
GML & Geography Markup Language & Geospatial data service \\
Common & OGC Web Services Common Specification & Geospatial data service \\
SLD & Styled Layer Descriptor & Geospatial data service \\
WCS & Web Coverage Service & Geospatial data service \\
WFS & Web Feature Service & Geospatial data service \\
WMC & Web Map Context Documents & Geospatial data service \\
WMS & Web Map Service & Geospatial data service \\
\hline
\end{tabular}




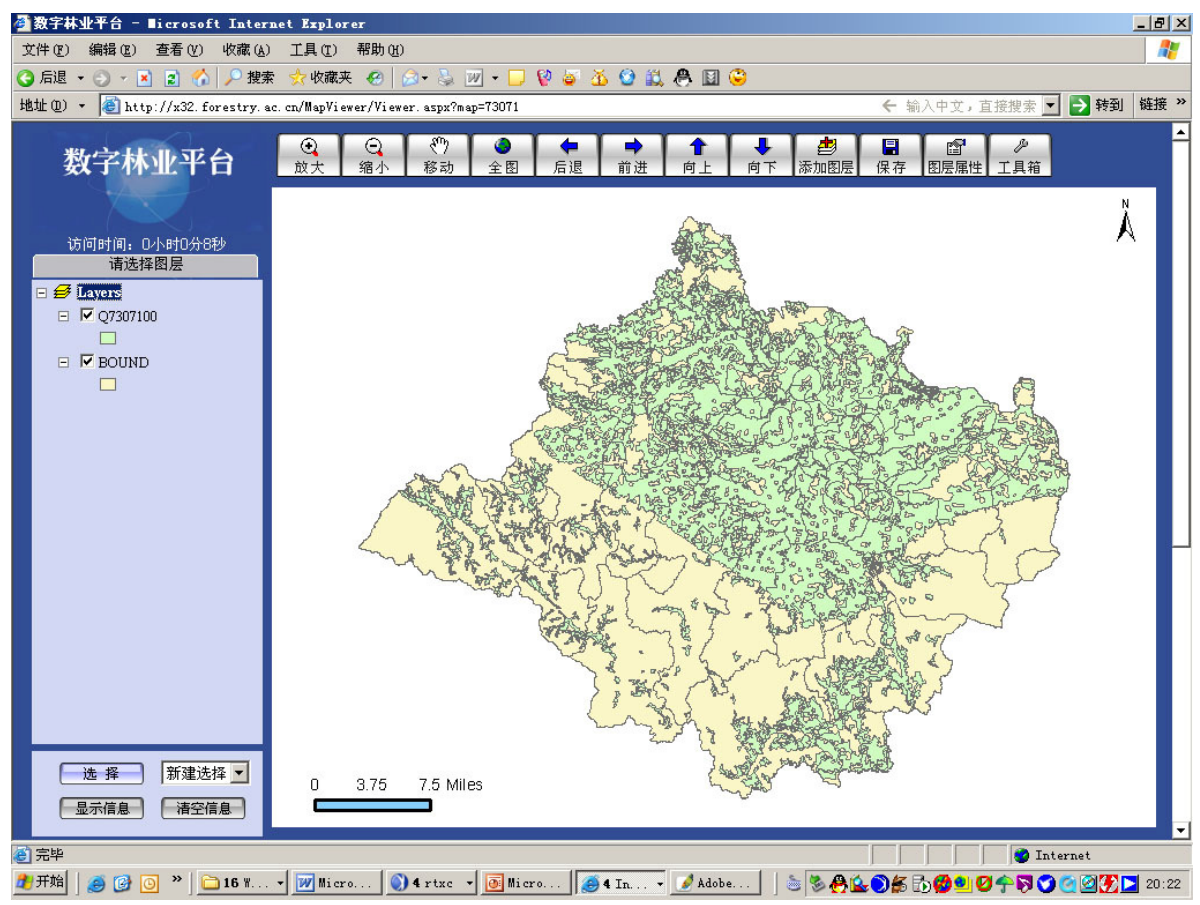

Fig. 2. A Web Feature service result presentation of digital forestry grid

\section{DISCUSSION AND CONCLUSION}

From above, we give a short presentation of the design of geospatial computational grid in the view of interoperability. The importance of the interoperable features in GCG cannot be ignored in the digital forestry system. Agents can be used to integrate the web service. But more key technologies are not put up in this paper, which is for further research.

\section{ACKNOWLEDGEMENTS}

This study has been funded by China National Key Research Project (863) "Research on the Web based Spatial Computation Service" (Contract Number: 2006AA12Z203) and "Research on the distributed management technique of the forest farm faced forest resources heterogeneous data" (Contract Number: 2006AA10Z240). 


\section{REFERENCES}

Aktas, M., Aydin, G., Donnellan, A., Fox, G., Granat, R., Grant, L., Lyzenga, G., McLeod, D., Pallickara, S., Parker, J., Pierce, M., Rundle, J., Sayar, A., and Tullis, T., Iservo 2004. Implementing the International Solid Earth Research Virtual Observatory by Integrating Computational Grid and Geographical Information Web Services Technical Report, Special Issue of Pure and Applied Geophysics (PAGEOPH) for Beijing ACES Meeting July, December.

Booth, D., Haas, H., McCabe, F., Newcomer, E., Champion, M., Ferris, C., and Orchard, D. 2004. "Web Services Architecture." W3C Working Group Note, 11 February 2004, Available from http://www.w3.org/TR/2004/NOTE-ws-arch-20040211/.

Brunett, S. 2001. Application experiences with the Globus Toolkit, Proceedings of the 7th IEEE Symposium on High Performance Distributed Computing. IEEE Press: 81-89.

Chen Aijun, Di Liping, Wei Yaxing, Liu Yang, Bai Yuqi 2005. Grid Computing Enabled Geospatial Catalague Web Service, SPRS 2005 Annual Conference Baltimore, Maryland March: 7-11.

Cox, S., Daisey, P., Lake, R., Portele, C., and Whiteside, A. (eds) 2003 OpenGIS Geography Markup Language (GML) Implementation Specification. OpenGIS project.

Foster, I., Kesselman, C., and Tuecke, S. 2001. The Anatomy of the Grid: Enabling Scalable Virtual Organizations, International J. Supercomputer Applications 15(3).

Karl Czajkowski, Donald F. Ferguson, Ian Foster, Jeffrey Frey, Steve Graham, Tom Maguire, David Snelling, Steve Tuecke. 2004. From Open Grid Service Infrastructure to WSResource Framework: Refactoring \& Evolution Version 1.0.Globus Alliance.

Li Guoqing, Liu Dingsheng, Sun Yi. 2005. A Grid Research on desktop type software for spatial information processing.

Moore, R., Baru, C., Marciano, R., Rajasekar, A., and Wan, M. 1999. Data-intensive computing, in Foster, I. and Kesselman, C. (eds) The Grid: Blueprint for a New Computing Infrastructure, Morgan Kaufmann: 105-129.

The Open Geospatial Consortium (OGC): http://www.opengeospatial.org

Vretanos, P. (ed.) 2002. Web Feature Service Implementation Specification, OpenGIS project document: OGC 02-058, version 1.0.0. 